\title{
(2) OPEN ACCESS \\ Fifteen-minute consultation: Kawasaki disease: how to distinguish from other febrile illnesses: tricks and tips
}

\author{
Alison Kelly, ${ }^{1}$ Katie Sales, ${ }^{2}$ Mary Fenton-Jones, ${ }^{2}$ Robert Tulloh (i) ${ }^{3}$
}

\begin{abstract}
'Paediatric Rheumatology, Bristol Royal Hospital for Children, Bristol, UK ${ }^{2}$ Bristol Royal Hospital for Children, Bristol, UK ${ }^{3}$ Paediatric Cardiology, Bristol Royal Hospital For Children, Bristol, UK
\end{abstract}

\section{Correspondence to} Dr Alison Kelly, Paediatric Rheumatology, Bristol Royal Hospital for Children, Bristol BS2 8BJ, UK; Alison.Kelly@ UHBristol.nhs.uk

Received 13 March 2019 Revised 8 July 2019 Accepted 16 August 2019 Published Online First 3 January 2020

\section{Check for updates}

(c) Author(s) (or their employer(s)) 2020. Re-use permitted under CC BY-NC. No commercial re-use. See rights and permissions. Published by BMJ.

To cite: Kelly A, Sales $K$, Fenton-Jones M, et al. Arch Dis Child Educ Pract Ed

2020;105:152-156.

\begin{abstract}
Kawasaki disease (KD) is challenging to diagnose because there is no specific laboratory test and the presentation is often similar to common childhood infections. We highlight some of those KD diagnostic challenges. KD, a self-limiting vasculitis, can cause coronary artery aneurysms. The aim is to optimise management during the acute febrile illness to try and prevent these because a giant coronary artery aneurysm is devastating enough without thinking that it might have been prevented. The conundrum for acute paediatricians is which clinical features best distinguish the febrile child with possible $K D$, needing intravenous immunoglobulin, from the many other children with febrile illnesses.
\end{abstract}

\section{BACKGROUND}

Kawasaki disease (KD), a self-limiting systemic vasculitis, remains of unknown aetiology and can cause irreversible coronary artery aneurysms (CAAs). In the absence of a pathognomonic test, diagnosis relies on the clinical presentation. ${ }^{1}$ The diagnosis is made in the presence of prolonged fever of 5 days or more combined with four of the five clinical criteria (see box 1). With fewer than four criteria present, the diagnosis of incomplete $\mathrm{KD}$ is considered taking into account laboratory and echocardiographic results. It is becoming more common, with incidence doubling every 10 years. ${ }^{2}$ Now around 300 children have KD each year in the UK (about 10 per 100000 children per year under 5) and may have a lifetime of consequences if not treated promptly. ${ }^{3}$

The decision of when to give intravenous immunoglobulin (IVIG) relies on clinical experience and skilled decision making, taking into account which clinical features fit with a diagnosis of KD. One gram of IVIG is derived from the combined plasma of $>1000$ donors. $^{4}$ Donors are screened for known infections, for example, hepatitis B; however, there remains a theoretical risk of infections, such as those yet to be discovered. IVIG side effects include fever, headache, vomiting, joint pain, aseptic meningitis, and very rarely a severe allergic reaction. Overdiagnosis of $\mathrm{KD}$ has recently been reported in the USA. ${ }^{5}$ The challenge faced by acute general paediatricians is to prevent CAAs while not overdiagnosing.

\section{CONUNDRUMS WHEN DIAGNOSING \\ KD}

The child with features that resemble an infection but also fulfils some criteria for KD A 4-year-old child is reviewed on the acute ward round having presented overnight to the emergency department (ED). The fever has been present for 4 days with cervical lymphadenopathy, a red oropharynx and rash. Antibiotics have not been started. The ED doctor has written in the notes that KD has been considered,

Box 1 Clinical diagnosis of Kawasaki disease

Fever for 5 days or more, plus four of the following five criteria (NB: can be diagnosed at 4 days if all five criteria present, or incomplete with fewer criteria):

- Red rash (any type of rash and often from day 1 , but may disappear).

- Red conjunctival injection (not purulent or sticky).

- Red lips and mouth, cracked dry lips.

- Red hands and feet (peeling comes late after 10 days - too late to prevent aneurysms).

- Lymphadenopathy (typically a single large lymph node in the neck). 
Box 2 Other recognised findings in Kawasaki disease

Extreme irritability.

Aseptic meningitis.

- Arthralgia and arthritis.

- Pancarditis.

- Pericardial effusions.

- Congestive cardiac failure.

- Valvular dysfunction.

- Coronary artery abnormalities.

- Diarrhoea, vomiting and abdominal pain.

- Hepatic dysfunction.

- Hydrops of the gall bladder.

- Erythema and induration at the BCG site.

- Testicular swelling.

- Anterior uveitis.

- Perineal erythema and desquamation.

but because only three of the five criteria were present IVIG was not started.

This may be an acute infection, such as a betahaemolytic streptococcal or adenoviral tonsillitis. However, should IVIG be given now? It would seem sensible in many similar cases of possible KD to try intravenous broad-spectrum antibiotics for potential bacterial illness for 24 hours while observing, and to avoid exposure to IVIG unless fever and features of KD continue. Viral throat swabs may help to identify a pathogen, but with the aetiology of KD being unknown there also remains the possibility that KD may be triggered by, or coexist with, an infection. A detailed history should ask specifically for KD criteria, since they often occur sequentially. Photographs taken by parents earlier in the illness may also help to confirm a diagnosis. Considering KD early in the admission is important. Not thinking of KD may lead to delayed treatment or missed cases.

Diagnosis may be helped if a typical feature of KD is present, such as irritability. KD can cause a wide variety of non-specific symptoms and signs, for example gastrointestinal symptoms, in addition to the criteria. Some findings which can help to suggest KD include reactivation of BCG immunisation site, irritability,

\section{Box 3 Results suggesting severe Kawasaki disease}

- High white cell count.

- Very high C reactive protein.

L Low serum sodium.

- Low albumin.

- Low haemoglobin.

- Low platelet count.

- High aspartate transaminase.

- High bilirubin.

- High ferritin (NB: very high ferritin, $>10000 \mu \mathrm{g} / \mathrm{L}$, indicates haemophagocytic lymphocytic histiocytosis).
Box 4 Routine investigations in suspected

Kawasaki disease

Blood

- Inflammatory markers (erythrocyte sedimentation rate and $\mathrm{C}$ reactive protein).

- Liver function tests.

- Full blood count, urea and electrolytes, ferritin, clotting and blood cultures.

- Antistreptolysin titre (ASOT) and anti-DNAse B, Ddimer, mycoplasma serology, autoimmune profile, ANCA, C3 and C4, and save serum.

- Viral serology/PCR.

- Urine dip and. urine microscopy, culture and sensitivity

- Throat swab (bacterial and viral).

- Echocardiogram

- At diagnosis, 2 weeks and 6 weeks.

ECG

perineal redness, meningeal irritation and arthritis (see box 2). KD irritability may persist despite antipyretics and is extreme compared with many other illnesses.

Clearly every febrile child with red oropharynx and lymphadenopathy does not require IVIG, and deciding on treatment relies entirely on skilled judgement of whether clinical features fit with a diagnosis of KD. The only pathognomonic test is CAA on echocardiogram, which is what early IVIG treatment would hopefully prevent.

\section{The persistently febrile very young child}

A 4-month-old presented with 3 days of diarrhoea and a blanching rash. His parents had not noticed fever until vomiting prompted attendance to hospital. On arrival he was feverish $\left(38^{\circ} \mathrm{C}\right)$, and the following day the rash resolved. Investigations show raised $\mathrm{C}$ reactive protein (CRP) and negative septic screen. Despite ceftriaxone intermittent fevers continue. On day 8 of fever in hospital, an echocardiogram shows giant CAAs. Treatment is started including IVIG, steroids due to a lack of response to IVIG, and heparin.

Features of KD such as rash may resolve during the illness, and very young children may have incomplete $\mathrm{KD}$ and few features of KD except persistent fever. Very young children are high risk as they are less likely to respond to IVIG, are more likely to require steroids and are more likely to develop CAAs. Try not to leave a very young child without treatment for KD if the febrile illness is prolonged. Less than 6 months old is a red flag for KD complications; these children have the fewest features of KD but develop the worst coronary artery disease. In the UK 39\% of infants develop coronary artery involvement, highlighting the need to recognise KD and treat urgently. ${ }^{3}$

The febrile child with gastrointestinal symptoms

A 3-year-old is reviewed on the ward. He was admitted 3 days ago with lethargy, diarrhoea and a persistent 
fever not responding to antipyretics. Investigations have shown raised inflammatory markers, low albumin and distended gall bladder on abdominal ultrasound. His parents report today is day 7 of fever. Extreme irritability and cervical lymphadenopathy, along with a new finding of injected conjunctivae, are noted on review. KD is diagnosed and IVIG given.

A fever unresponsive to antipyretics or disproportionate irritability can be indicators of KD. Viral-like symptoms such as sore throat, abdominal pain, diarrhoea or cough are not reassuring because these can occur in KD. Features may appear sequentially and may not be present together on examination. Children with unexplained fever require repeat reviews for any developing features of $\mathrm{KD}$. A child not following the normal trajectory of illness, in this case not typical for viral gastroenteritis, needs to have other conditions, such as KD, considered as early as possible. Gallbladder distension can occur in $\mathrm{KD}$ and raises the possibility of abdominal ultrasound being a useful investigation when a diagnosis is being considered.

\section{The child treated with IVIG then re-presents febrile}

A 1-year-old is diagnosed with complete KD and has a normal echocardiogram. She becomes afebrile during the IVIG and is discharged the following day. She is readmitted to hospital 3 days later with a further history of fevers at home since discharge. The echocardiogram shows small CAAs and she receives a second dose of IVIG and steroids.

Children are routinely monitored in hospital following treatment for $\mathrm{KD}$ to ensure that they do not have recrudescence of fever, which requires further treatment. Observation for at least 48 hours without fever is reassuring following treatment. This child has aneurysms in keeping with her KD diagnosis. However, in other cases if there is a lack of sustained response to IVIG, consideration of other diagnoses would be appropriate, for example, systemic juvenile idiopathic arthritis, and discussion with paediatric rheumatologists is advised as steroid therapy might be indicated.

\section{The child who peels or has a retrospective diagnosis of KD}

A 6-year-old re-presents to ED with peeling fingers 10 days after resolution of an 8-day febrile illness. Her parents read on the internet about KD and are concerned that she may need IVIG. Inflammatory markers, ECG and echocardiogram are normal.

KD remains one possible differential diagnosis retrospectively. However there is no evidence of ongoing inflammation and no CAAs, so the child is started on low-dose aspirin and an appointment for a 6 -week echocardiogram is made. It is not uncommon

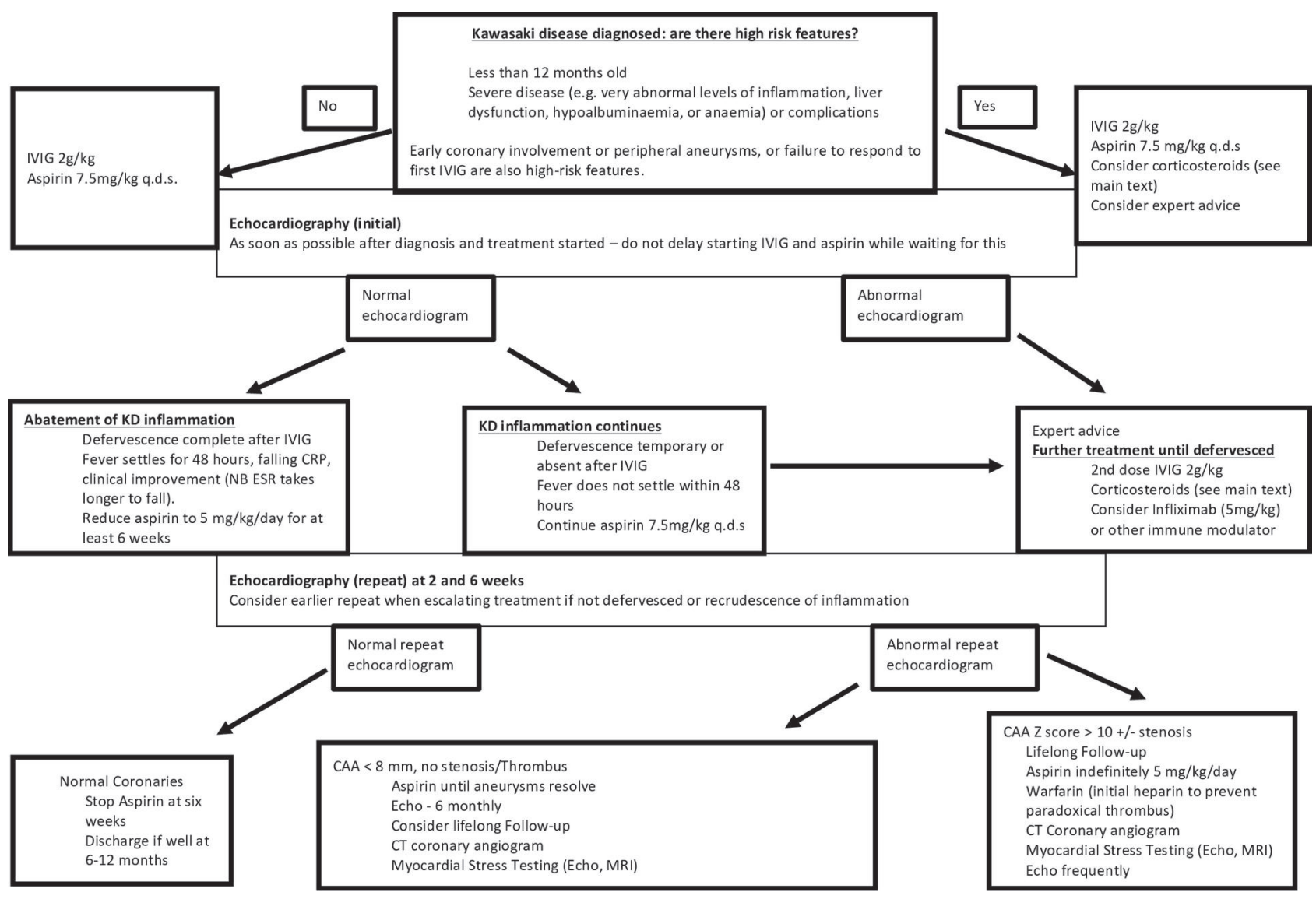

Figure 1 Algorithm for management of KD. CAA, coronary artery aneurysm; CRP, C reactive protein; ESR, erythrocyte sedimentation rate; IVIG, intravenous immunoglobulin; KD, Kawasaki disease; q.d.s, four times a day. 


\section{Test your knowledge}

1. Kawasaki disease is increasingly common. The current incidence per year in the UK in children under 5 years is (single best answer):
A. 1 in 100000
B. 1 in 50000 .
C. 1 in 30000
D. 1 in 10000 .
E. 1 in 1000 .

2. Kawasaki disease symptoms can include (multiple answers):
A. Arthritis.
B. Irritability.
C. Dysuria.
D. Gastrointestinal symptoms.
E. Meningism.

3. The frequency of infants (those under 1 year) having coronary artery involvement in the UK in Kawasaki disease after diagnosis and treatment is (single best answer):
A. $1 \%$.
B. $4.7 \%$.
C. $17 \%$
D. $25 \%$.
E. $39 \%$

4. The correct treatment regimen for acute Kawasaki disease is (multiple answers):

A. $1 \mathrm{~g} / \mathrm{kg}$ immunoglobulin over 6 hours and repeated the next day.

B. $2 \mathrm{~g} / \mathrm{kg}$ immunoglobulin over 12 hours plus 7.5 mg aspirin 4 times a day.

C. Immunoglobulin infusion should be repeated at 48 hours if there is no resolution of fever.

D. Intravenous methylprednisolone should be added to the initial treatment in children with high-risk features of age $<6$ months, CRP $>100$, low platelet count and coronary artery dilation already present.

E. Infliximab is commonly given to suppress coronary artery aneurysm formation.

5. Transition to adult care is important in all chronic diseases (multiple answers).

A. Kawasaki disease is not chronic, so no transition is needed.

B. All children who have had Kawasaki disease need long-term care.

C. Those who have giant coronary artery aneurysms are at most risk and have to have annual assessment, ongoing investigations and transition to adult Kawasaki clinics.

D. Those whose aneurysms have resolved on imaging can be discharged.

E. $3.8 \%$ of adults under 50 years old undergoing coronary angiography have aneurysms and ST elevation myocardial infarction that are due to previous Kawasaki disease.

Answers to the quiz are at the end of the references. for parents to suggest the diagnosis of $\mathrm{KD}$ and cases should be reviewed accordingly.

The child reviewed in primary care with $\mathbf{4}$ days of fever A 7-year-old is seen in primary care with 4 days of fever. A rash on day 2 has resolved and he had a cough at the beginning of the illness. His temperature returns to normal with paracetamol. He has a red oropharynx, runny nose, no tonsillar exudate and no lymphadenopathy. The doctor documents absence of other features of KD.

This child is approaching day 5 of fever; however, this is probably a common upper respiratory tract infection. Advice should be given to his family to return for medical review in 2 days if his fever continues. If all the diagnostic features of complete KD were present, it would be acceptable to treat at fewer than 5 days of fever.

\section{KD INVESTIGATIONS AND SCORING SYSTEMS}

Investigations can help to diagnose $\mathrm{KD}$, for example, raised CRP and erythrocyte sedimentation rate, despite there not being a pathognomonic test. Some patterns of results suggest severe KD (see box 3 ).

Viral tests or throat swabs do not help exclude KD. Lumbar puncture may be positive for non-specific inflammation (lymphocytes) in KD. Urine may show sterile pyuria. Abdominal ultrasound may show a swollen gall bladder if performed. Some investigations are routine in suspected KD (box 4).

There are scoring systems used in Japan to detect those at high risk of not responding to treatment, but they are not reliable in the UK. ${ }^{6}$

\section{WHEN SHOULD I USE AN ECHOCARDIOGRAM TO HELP ME MAKE THE DIAGNOSIS?}

An echocardiogram should be performed on the same day as the diagnosis is made. It can help make the diagnosis if abnormal, but a normal echocardiogram does not exclude KD. If a child presents after resolution of the febrile illness and is suspected on history to have had KD, they may have the diagnosis confirmed if the echocardiogram is abnormal.

The size of the coronary arteries is assessed by $\mathrm{Z}$ score (the number of SD above average for that sized child). A Z score equal to or more than 2.5 at 6 weeks is a CAA. If the child has a giant CAA ( $\mathrm{Z}$ score $>10$ ), then the early management will be different (see figure 1). Some children can have pericardial effusion, depressed heart function or valve regurgitation, and so it is important to know about this early.

\section{TREATMENT}

It is important that IVIG $(2 \mathrm{~g} / \mathrm{kg}$ over approximately 12 hours) is given at the earliest opportunity (figure 1). Every day's delay markedly increases the risk of CAA formation. High-dose aspirin, as an anti-inflammatory, should be given at the same time $(7.5 \mathrm{mg} / \mathrm{kg} 6$ hourly) 
until apyrexial for 48 hours. Those children who are high risk (as above) should receive intravenous steroids in addition (discuss with local expert).

If the fever has settled for 48 hours, then the child can be sent home on low-dose aspirin $(5 \mathrm{mg} / \mathrm{kg}$ each day) for its antiplatelet effect. If it does not settle, they may need another dose of immunoglobulin, steroids considering, and a plan made for a biologic if the fever persists, such as infliximab or anakinra. However, all these children who are difficult to manage should be discussed with an expert in KD.

\section{FOLLOW-UP}

Children who never had coronary artery involvement can be discharged at 6 months, but those with aneurysms are followed lifelong and stay on low-dose aspirin, even if they resolved (see figure 1). Those under follow-up should be transitioned to adult KD clinics and have a risk of major coronary events in the future (30\% in those with giant aneurysms). Of young adults with myocardial ischaemia, $3.8 \%$ have been found to have CAA on angiography.

\section{PATIENT-SPECIFIC PROTOCOLS: WHAT ARE THEY?}

As a result of a patient safety alert issued by NHS England 3 years ago, all children with known cardiac complications of KD should be issued with a patientspecific protocol. This gives a brief summary of the child's complications and what to do in the event of acute cardiac symptoms, when they should usually be taken direct to a specialist children cardiac surgical centre for acute management, such as thrombolysis of the coronary arteries or cardiac catheter lab intervention.

\section{FUTURE DIRECTIONS}

It is hoped that there will be a diagnostic test in the near future. KD often remains a challenging diagnosis to make with certainty. Increasing consultation with colleagues with expertise in KD may help to inform clinical decision making.

\section{Competing interests None declared.}

Patient consent for publication Not required.

Provenance and peer review Commissioned; externally peer reviewed.

Open access This is an open access article distributed in accordance with the Creative Commons Attribution Non Commercial (CC BY-NC 4.0) license, which permits others to distribute, remix, adapt, build upon this work noncommercially, and license their derivative works on different terms, provided the original work is properly cited, appropriate credit is given, any changes made indicated, and the use is non-commercial. See: http://creativecommons.org/ licenses/by-nc/4.0/.

ORCID iD

Robert Tulloh http://orcid.org/0000-0002-3180-6993

\section{REFERENCES}

1. Eleftheriou D, Levin M, Shingadia D, et al. Management of Kawasaki disease. Arch Dis Child 2014;99:74-83.

2. McCrindle BW, Rowley AH, Newburger JW, et al. Diagnosis, treatment, and long-term management of Kawasaki disease: a scientific statement for health professionals from the American heart association. Circulation 2017;135:e927-99.

3. Tulloh RMR, Mayon-White R, Harnden A, et al. Kawasaki disease: a prospective population survey in the UK and Ireland from 2013 to 2015. Arch Dis Child 2019;104:640-6.

4. British National formulary, BNF, NICE, 2018. Available: https:// bnf.nice.org.uk/treatment-summary/immunoglobulins.html

5. Coon ER, Wilkes J, Bratton SL, et al. Paediatric overdiagnosis modelled by coronary abnormality trends in Kawasaki disease. Arch Dis Child 2018;103:937-41.

6. Davies S, Sutton N, Blackstock S, et al. Predicting IVIG resistance in UK Kawasaki disease. Arch Dis Child 2015;100:366-8.

7. Waterhouse BR, Tulloh RMR, Kim Y, et al. Retrospective study of the impact of unrecognised Kawasaki disease, coronary aneurysm and ectasia. Int J Cardiol 2017;248:30813.

\section{Answers to the multiple choice questions}

1. D is true. In Japan, it is now up to 3.2 per 1000 children such that the prevalence is over $10 \%$.

2. $A, B, D$ and $E$ are true. KD can cause almost any symptom; however, while sterile pyuria does occur, dysuria is not reported.

3. $E$ is true. The incidence of coronary artery involvement is very high in treated infants. These children often only have some of the features of Kawasaki disease and can easily be missed due to incomplete Kawasaki disease. They have the worst cardiac involvement.

4. B, C and D are true. Infliximab is a good agent for treating resistant Kawasaki disease but is only used rarely and in resistant cases where immunoglobulin, aspirin and intravenous steroids have failed to work.

5. $C$ and $E$ are true. We do not follow up children who have never had coronary artery involvement; it would cause unnecessary anxiety and there is no evidence that they are at risk. Those with severe coronary artery disease should be followed very carefully, and $3.8 \%$ of adults undergoing coronary angiography have artery aneurysms due to previous Kawasaki disease. 